\title{
Concepciones iniciales sobre la Primera Ley de la Termodinámica a través de la implementación de un proyecto
}

\section{The learning of the First Law of Thermodynamics through the implementation of a project}

\author{
Karen Lucia Lamar Rodriguez ${ }^{1}$ \\ Maria Teresa Bolivar Torres ${ }^{2}$
}

\section{Resumen}

En este artículo se presenta una propuesta que permite abordar la primera ley de la termodinámica a través de la estrategia pedagógica Aprendizaje Basado en Proyectos (ABPy), con lo cual se busca promover un aprendizaje significativo crítico en 91 estudiantes del grado $8^{\circ}$ de la Institución Educativa Alfonso Upegui Orozco de Medellín, Colombia. Se exponen en este reporte algunos referentes teóricos y se describe la metodología de investigación y algunos asuntos de la estrategia de intervención. Este reporte hace énfasis, en algunos de los resultados obtenidos al aplicar un instrumento para indagar las ideas previas de los estudiantes sobre conceptos asociados a la enseñanza de la primera ley de la Termodinámica. Finalmente, se realizan algunas discusiones sobre los resultados y se establecen algunas contrastaciones con base en la literatura revisada.

${ }^{1}$ Karen Lucia Lamar Rodriguez. Licenciatura en Educación Básica en Ciencias Naturales y Educación Ambiental de la Universidad de Antioquia, Medellín, Colombia. Actualmente, cursando la práctica pedagógica II, en la línea de investigación Aprendizaje Basado en Proyectos (ABPy). Correo electrónico karen.lamar@udea.edu.co

ID: http://orcid.org/0000-0002-6114-4767

2 Maria Teresa Bolivar Torres. Licenciatura en Educación Básica en Ciencias Naturales y Educación Ambiental de la Universidad de Antioquia, Medellín, Colombia. Actualmente, cursando la práctica pedagógica II, en la línea de investigación Aprendizaje Basado en Proyectos (ABPy). Correo electrónico: teresa.bolivar@udea.edu.co

ID: https://orcid.org/0000-0002-5541-7757 
RECIE. Revista Electrónica Científica de Investigación Educativa

Vol. 4, núm. 2, enero-diciembre 2019, pp. 971-982.

\title{
Palabras claves
}

Aprendizaje significativo crítico, Aprendizaje basado en proyectos, enseñanza de la física.

\begin{abstract}
This article presents a proposal that allows to address the first law of thermodynamics through the pedagogical strategy Project Based Learning (PjBL), which seeks to promote critical meaningful learning in 91 students of 8th grade of the Institución Educativa Alfonso Upegui Orozco in Medellín, Colombia. Some theoretical referents are exposed in this report and the research methodology and also, some issues of the intervention strategy are described. This report emphasizes, in some of the results obtained by applying an instrument to investigate the previous ideas of students about concepts associated with the teaching of the first law of Thermodynamics. Finally, there are some discussions about the results and some answers were compared based on the literature reviewed.
\end{abstract}

\section{Keywords}

Critical meaningful learning, project-based learning, physics teaching.

\section{Introducción}

A través de la historia, las sociedades han sufrido una serie de cambios y transformaciones tanto industriales como tecnológicas, en las cuales la educación ha tenido un papel fundamental. Sin embargo, la educación que se ha realizado hasta este momento es limitada y estática, ya que, tras años de enseñanza, la mayoría de las clases magistrales son alejadas del contexto y de las necesidades del estudiante, sin tener en cuenta que estamos ante una sociedad de la información y que por ello se necesita de una educación acorde a sus necesidades. Tal como lo propone Leymonié (2009):

La concepción de la enseñanza y del aprendizaje han sufrido cambios significativos en los últimos años, con importantes consecuencias sobre la manera de entender cómo los estudiantes aprenden y, por lo tanto, sobre las posibles metodologías a desarrollar en las aulas. Estos cambios van de la mano con las nuevas concepciones de la ciencia y, por lo tanto, de educación científica (p.12).

Por otro lado, la enseñanza de la física, especialmente de los conceptos asociados a la primera ley de la termodinámica tales como calor, temperatura y trabajo, entre otros, son enseñados, en su mayoría, bajo el método tradicional de forma desarticulada, lo que imposibilita un 
aprendizaje significativo y una relación directa con las actividades diarias de los estudiantes, es por ello que se hace necesaria la incorporación de otras alternativas de enseñanza.

En este orden de ideas, al revisar el plan de área de ciencias naturales 2012-2017 de la Institución Educativa Alfonso Upegui Orozco, no se encuentra planteada la enseñanza de la termodinámica para el grado octavo, pero se realiza una aproximación de este tema en el grado $11^{\circ}$ desde la noción de gases y conversión de unidades de temperatura en el grado $9^{\circ}$, dejando por fuera un tema muy importante para la explicación de situaciones cotidianas tales como ¿por qué sudamos cuando corremos o por qué sentimos calor y frío?, y que de acuerdo con los Derechos Básicos de Aprendizaje (DBA) se debe enseñar en el grado 8. Lo anterior causa en los estudiantes un vacío conceptual en física, lo que ocasiona una resistencia para la comprensión de los conceptos asociados a la termodinámica.

De acuerdo a lo anterior, una de las estrategias pedagógicas propuestas para generar un cambio significativo en los métodos de enseñanza es el Aprendizaje Basado en Proyectos (ABPy), el cual se caracteriza porque permite un cambio en la cultura escolar, fortaleciendo las relaciones maestro-estudiante, maestro-maestro, estudiante-estudiante y en donde el docente pasa de ser el que transmite el conocimiento a ser un mediador, y en la misma medida, el estudiante deja de ser el que recibe la teoría para convertirse en el propio constructor de su conocimiento, bajo una atmósfera de cooperación y democracia entre los pares, como dice García \& Basilotta (2016) "este método promueve el aprendizaje individual y autónomo dentro de un plan de trabajo definido por objetivos y procedimientos. Los alumnos se responsabilizan de su propio aprendizaje, descubren sus preferencias y estrategias en el proceso" (p.114).

Así mismo, el rol que debe ocupar el estudiante es el de un sujeto crítico, autónomo y reflexivo que asuma su proceso de aprendizaje partiendo del querer hacer, es decir, son ellos quienes en gran medida deciden qué aprender y con qué fin lo hacen, lo que permite que el ABPy sea un detonante para el aprendizaje significativo. En este sentido, en esta investigación se planteó como objetivo general, analizar el aprendizaje que logran los estudiantes de grado octavo sobre la primera ley de la termodinámica a través de la estrategia pedagógica Aprendizaje Basado en Proyectos (ABPy).

\section{Marco referencial}

Para poder desarrollar esta investigación se hace necesario definir una base teórica que permita sustentar las decisiones que se tomen durante la 
implementación del proyecto y el análisis de la información. En este sentido, se presentan algunos referentes en relación con el Aprendizaje Basado en Proyectos (ABPy) y el Aprendizaje Significativo Crítico.

\section{Aprendizaje Basado en Proyectos (ABPy)}

El aprendizaje Basado en Proyectos es una propuesta innovadora que busca integrar al estudiante en la construcción de su conocimiento, de tal manera que éste pasa a ser el protagonista de su aprendizaje de forma autónoma y el docente se convierte en un facilitador. De acuerdo con Ciro (2012)

El Aprendizaje Basado en Proyectos (ABPy) es una alternativa formativa que trasciende los principios de la pedagogía activa, pues permite comprender el contexto real del desempeño profesional articulando conocimientos propios de la disciplina e intentando lograr un sinergismo que conduzca a una formación. (p.17)

Por su parte, esta estrategia tiene ocho características planteadas por Larmer, Mergendoller \& Boss en el 2015 y que hacen parte de la propuesta del Buck Institute for Education (BIE), las cuales se muestran en la figura 1.

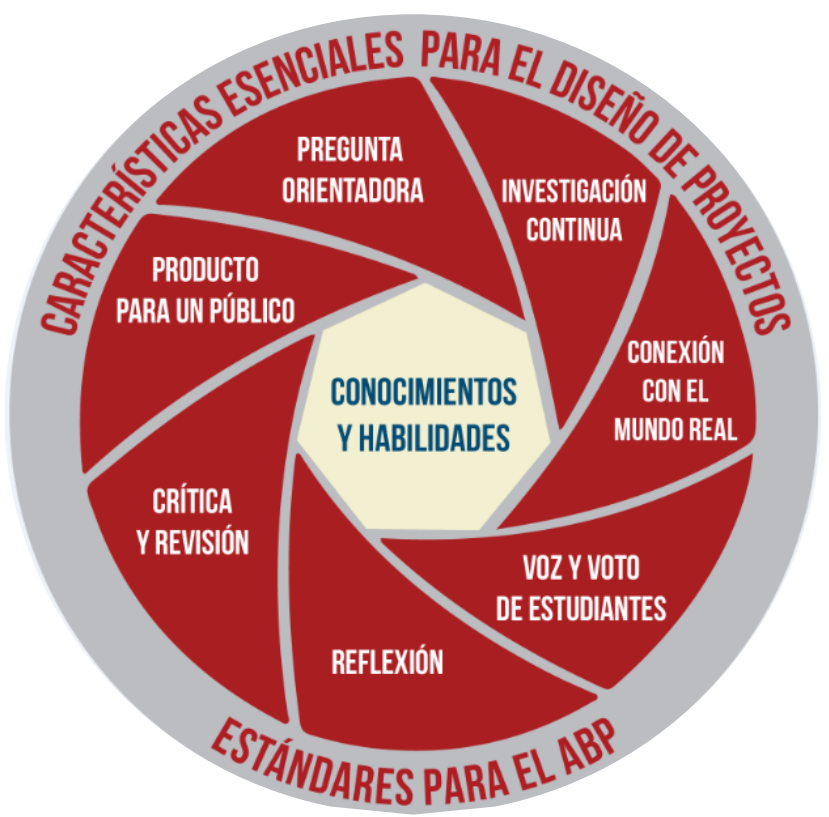

Figura 1: Tomado de "estándares de oro" - características esenciales para el diseño de proyectos, según el Buck Institute for Education (BIE). Larmer, Mergendoller \& Boss, (2015). 


\section{Teoría del Aprendizaje Significativo Crítico}

Partiendo de la idea de que el aprendizaje significativo se caracteriza por la interacción entre el nuevo conocimiento y el conocimiento previo y que este a su vez nos ayuda a formular conocimientos más estructurados, es importante mencionar lo que plantea Ausubel, (1976, 2002); Moreira, (1997).

El aprendizaje significativo es el proceso según el cual se relaciona un nuevo conocimiento o información con la estructura cognitiva del que aprende de forma no arbitraria y sustantiva o no literal. Esa interacción con la estructura cognitiva no se produce considerándola como un todo, sino con aspecto relevantes presentes en la misma, que reciben el nombre de subsumidores o ideas de anclaje.

Resulta oportuno así mismo, el aporte que nos presenta Moreira M, A (2005), sobre su visión crítica de la teoría de aprendizaje significativo, así:

[...] es aquella perspectiva que permite al sujeto formar parte de su cultura y, al mismo tiempo, estar fuera de ella." Se trata de una perspectiva antropológica en relación a las actividades de su grupo social, que permite al individuo participar de tales actividades, pero, al mismo tiempo, reconocer cuándo la realidad se está alejando tanto que ya no se está captando por parte del grupo (p.7).

Partiendo de la idea de generar un aprendizaje significativo, es preciso hacer la salvedad de que esta teoría se caracteriza porque el principal fin, es que el sujeto pueda comprender, representar y resolver situaciones propias de su vida y adoptar una postura crítica frente a todos los escenarios que se le puedan presentar. Además, hace alusión a once principios Principio del conocimiento previo, principio de la interacción social y del cuestionamiento, principio de la no centralización en el libro de texto, principio del aprendiz como perceptor/representador, principio del conocimiento como lenguaje, principio de la conciencia semántica, principio del aprendizaje por el error, principio del desaprendizaje, principio de incertidumbre del conocimiento, principio de la no utilización de la pizarra, de la participación activa del alumno, de la diversidad de estrategias de enseñanza y principio del abandono de la narrativa, de dejar que el alumno hable, propuestos por Moreira (2005).

Para este reporte, se hace énfasis en el principio del conocimiento previo el cual según Moreira (2005) es la variable más importante porque se aprende a partir de lo que ya se sabe. Para ser crítico de algún conocimiento, de algún concepto, de algún enunciado, en primer lugar, el 
sujeto tiene que aprenderlo significativamente y, para eso, su conocimiento previo es, aisladamente, la variable más importante.

Teniendo como base lo anterior y a partir de las evidencias de aprendizaje obtenidas, se realizará una contrastación de las mismas con cada uno de los principios, con el propósito de establecer si los conocimientos adquiridos por los estudiantes, además de ser significativos, resultan tener algunas características de criticidad, bajo los argumentos descritos en cada uno de los principios.

\section{Metodología de investigación}

El paradigma bajo el cual se abordó el presente trabajo fue de corte cualitativo. Además, se utilizó el estudio de caso porque de acuerdo con Martínez (2006) quien cita a Yin (1989), este método tiene unos rasgos distintivos, entre los cuales destacamos, de acuerdo a la pertinencia de nuestro trabajo, que permite estudiar temas poco abordados y con gran pertinencia con el entorno real de los participantes (p.23).

Como instrumentos para la recolección de la información, se utiliza para la fase presentada en este reporte, la bitácora y el instrumento de indagación de ideas previas, el primer instrumento permite recolectar información sobre el proceso que lleva cada grupo y el segundo, tiene como propósito reconocer las ideas previas de los estudiantes a partir de la aplicación de un cuestionario.

En la siguiente figura (2), adaptada de Jorrín (2016) en el modelo rayuela, se presentan los asuntos más relevantes de este método en el marco de esta investigación. 


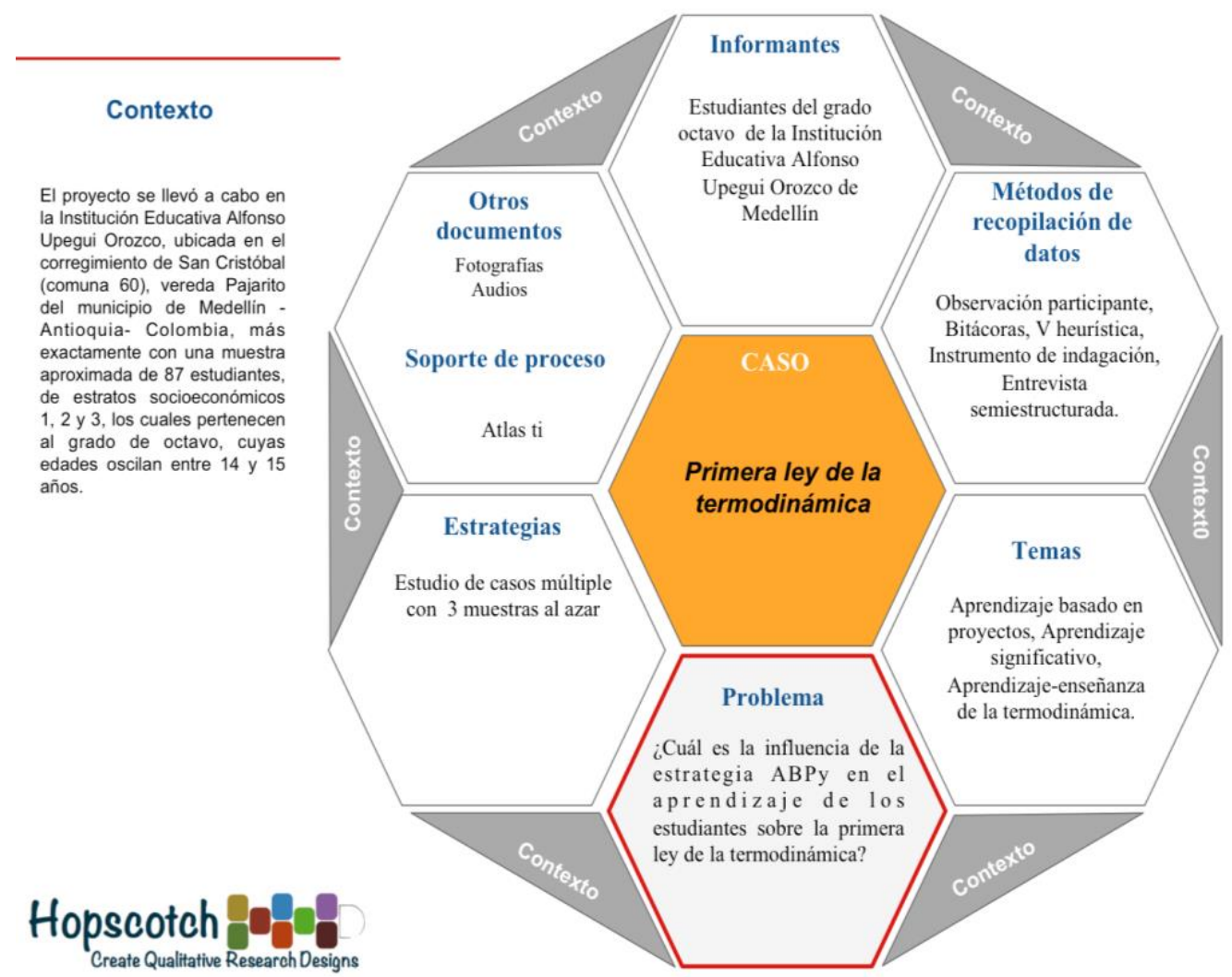

Figura 2. Componentes del estudio de caso. Método de rayuela. Jorrín (2016).

\section{Metodología de enseñanza}

Como metodología de enseñanza, se diseñó y aplicó un proyecto orientado por las características definidas por el Buck Institute for education (BIE), que se implementó para darle un orden y una estructura al proyecto, éste tuvo una duración de 10 semanas, las cuales están denominadas según el tema trabajado con los estudiantes.

También se hace necesario aclarar que el tiempo de intervención a los grupos es de 6 horas por semana. Para este reporte, se presentan los resultados parciales de la aplicación del instrumento de indagación. Se describen a continuación las tres etapas del proyecto, apertura (una semana), ejecución (ocho semanas) y cierre (una semana).

\section{Apertura del proyecto}

Durante esta fase, se dio a conocer a los estudiantes la pregunta orientadora del proyecto ¿cómo diseño y explico un ecodoméstico para el servicio de mi 
comunidad?, la metodología de trabajo, las áreas participantes (Ciencias Naturales, Tecnología, Ciencias Sociales, Lengua Castellana y Educación física) y el producto esperado (Ecodoméstico), además, se establecieron los grupos de trabajo, al interior de los cuales se asignaron un nombre y los roles de cada integrante, también, se trabajó en el diseño de una bitácora, en las cuales consignarán las reflexiones, datos, preguntas, de las actividades realizadas. Así mismo, se aplicó el instrumento de indagación de ideas previas.

\section{Ejecución}

Durante esta fase se aplicaron actividades para cumplir con el propósito de este trabajo, algunas de ellas fueron ¿Y qué hago con el calor?, en la cual se realizó una práctica de laboratorio que permitió a los estudiantes acercarse a los mecanismos de transferencia de calor y la aplicación en su contexto desde la utilización de materiales cotidiano. En la semana cuatro se realizó otra práctica de laboratorio virtual. En clase de ciencias se calculó la huella de carbono de cada estudiante. Otras actividades realizadas se denominaron ¿Y el calor de tu casa a dónde va? y "La energía de mi territorio" las cuales permitieron analizar ¿cómo influye la cantidad de electrodomésticos de la comunidad de Pajarito con el calentamiento global?

\section{Cierre}

En esta fase, los estudiantes presentaron los productos de su trabajo a la comunidad educativa, se realizaron procesos de autoevaluación y coevaluación.

\section{Resultados parciales}

Para la presentación de los resultados, se retoman las categorías propuestas en el instrumento de indagación de ideas previas (Ver tabla 3, columna 1) y se presenta el Caso 1, como objeto de análisis. Al tratarse de un estudio de Caso múltiple, se retoman las respuestas de 4 estudiantes. Aunque no todos respondieron a las preguntas propuestas. Posteriormente, se realizan algunos comentarios respecto a la información obtenida.

\begin{tabular}{|l|l|}
\hline $\begin{array}{l}\text { Definición } \\
\text { concepto } \\
\text { termodinámica }\end{array}$ & $\begin{array}{l}\text { E1 "Parte de la física que estudia la acción } \\
\text { mecánica del calor y las restantes formas de } \\
\text { energía" }\end{array}$ \\
& $\begin{array}{l}\text { E2 "Para mi es la función o manejo del frio y el } \\
\text { calor" }\end{array}$ \\
\hline
\end{tabular}




\begin{tabular}{|c|c|}
\hline & $\begin{array}{l}\text { - E3 "que la temperatura es como la acción del } \\
\text { calor y que forma energía" } \\
\text { - E4 "que puede pasar de frío a calor y de calor a } \\
\text { frio" }\end{array}$ \\
\hline $\begin{array}{l}\text { Relación de eventos } \\
\text { cotidianos con la } \\
\text { termodinámica }\end{array}$ & $\begin{array}{l}\text { El agua a temperatura ambiente } \\
\text { - E1"Porque hace parte de un cambio de } \\
\text { temperatura" } \\
\text { - E2 "porque tiene que ver con el frio entonces } \\
\text { hacer parte de la termodinámica" } \\
\text { - E3 "porque son los cambios de temperatura" }\end{array}$ \\
\hline Sistemas & $\begin{array}{l}\text { - Un termo con agua caliente: E1 "sistema } \\
\text { cerrado", E2 “No se" } \\
\text { - Un termómetro: E1 "sistema aislado", E4 "No } \\
\text { sé", E3 “sistema cerrado" }\end{array}$ \\
\hline $\begin{array}{l}\text { Explicación } \\
\text { fenómenos }\end{array}$ & $\begin{array}{l}\text { ¿por qué cuando estas en clase de educación física } \\
\text { sudas? } \\
\text { - E1"Porque el cuerpo sube la temperatura" } \\
\text { - E2 "no se" } \\
\text { - E3 "porque sube la temperatura a medida que } \\
\text { se hace ejercicio" }\end{array}$ \\
\hline $\begin{array}{l}\text { Temperatura } \\
\text { Calor }\end{array}$ & $\begin{array}{l}\text { - E1 “Obviamente la del hierro se iguala al frio del } \\
\text { aire acondicionado y la de madera conserva su } \\
\text { temperatura por su material. } \\
\text { - E2 "la mesa de hierro estaría más fría que la de } \\
\text { madera porque el hierro se enfría más que la de } \\
\text { madera. } \\
\text { - E3 "La mesa de hierro se pone muy fría por la } \\
\text { temperatura que tanto subió, y la mesa de } \\
\text { madera se pone fría pero no tanto como la de } \\
\text { hierro. }\end{array}$ \\
\hline $\begin{array}{l}\text { Transferencia de } \\
\text { energía en forma de } \\
\text { calor }\end{array}$ & $\begin{array}{l}\text { Cuando revuelves una sopa caliente con una } \\
\text { cuchara de metal, notas que la cuchara se calienta. } \\
\text { E2 "convección" } \\
\text { E3 "Conducción" } \\
\text { El sol está a } 150 \text { millones de kilómetros de la tierra. } \\
\text { Sin embargo, sentimos el calor de sus rayos en } \\
\text { nuestro planeta. } \\
\text { Tanto E2 como E3 dicen que es por "radiación" }\end{array}$ \\
\hline Máquinas y Trabajo & ¿explica que entiendes por \\
\hline
\end{tabular}

Concepciones iniciales sobre la Primera Ley de la Termodinámica... 
RECIE. Revista Electrónica Científica de Investigación Educativa Vol. 4, núm. 2, enero-diciembre 2019, pp. 971-982.

\begin{tabular}{|l|l|}
\hline trabajo? \\
E2 "que el calor hace trabajar una máquina para \\
poder que el barco se mueva" \\
E4 "no se"
\end{tabular}

Tabla 1. Respuestas asociadas a las categorías propuestas en el instrumento de indagación de ideas previas para el Caso 1.

Las respuestas descritas en la tabla 1, muestran en general un bajo conocimiento acerca del concepto termodinámica por parte de los estudiantes objeto de análisis. Esto se corresponde, con los hallazgos de Ramírez y Santana (2014) quien plantea que, para el caso del calor, este se asocia con temperaturas altas, se cree que las cosas tienen calor, como si se tratara de una sustancia en sí misma.

Asi mismo, Castiñeiras, De pro Bueno y Fernández (1998) en su investigación titulada Las partículas de la materia y su utilización en el campo conceptual de calor y temperatura: un estudio transversal, encontró que para los estudiantes la "Temperatura $=$ calor. Temperatura y calor son sinónimos y aquélla, en todo caso, mide la cantidad de calor que tiene el sistema. La temperatura depende de la masa o del volumen." (p. 462), esto mismo pareciera estar presente en las respuestas de los estudiantes, los cuales utilizan en algunos casos de manera indistinta ambos conceptos para dirigirse a los mismos fenómenos. Para estos mismos conceptos, Ramírez y Santana (2014), menciona que generalmente, la temperatura es equiparable al calor en la mayoría de los casos (p.78).

Además, cuando se solicita a los estudiantes relacionar los conceptos propuestos con eventos cotidianos o con base en conceptos más abstractos como maquina o trabajo, las respuestas son limitadas o nulas.

\section{Consideraciones finales}

Revisado el instrumento de indagación de ideas previas, se evidencia que, a nivel general, los estudiantes objeto de análisis presentan dificultades para comprender conceptos asociados a la termodinámica, como los mecanismos de transferencia de energía, sistemas; además se evidencia la confusión entre calor y temperatura al asumirlas como iguales.

Por otro lado, la información obtenida, sirvió como punto de partida para la elaboración de las actividades del proyecto, esto para ser coherentes con el principio del conocimiento previo y poder generar material que sea potencialmente significativo. 
Finalmente, este trabajo se encuentra en fase de análisis de la información, ya se han implementado las fases II y III del proyecto y se espera que, a la luz de la información obtenida, se puedan encontrar evidencias de aprendizaje que apunten a los principios propuestos por Moreira (2005) en su Teoría del Aprendizaje Significativo Crítico.

\section{Referencias}

Ausubel, D. (1976). Psicología educativa. Un punto de vista cognoscitivo. Ed. Trillas. México.

Ausubel, D. (2002). Adquisición y retención del conocimiento. Una perspectiva cognitiva. Ed. Paidós.

Castiñeiras, J., de Pro Bueno, A., \& Fernández, E. (1998). Las partículas de la materia y su utilización en el campo conceptual de calor y temperatura: un estudio transversal. Enseñanza de las ciencias: revista de investigación y experiencias didácticas, 16(3), 461-476.

Ciro, C. (2012). Aprendizaje Basado En Proyectos como estrategia de enseñanza y aprendizaje en la educación básica y media. Recuperado de http://www.bdigital.unal.edu.co/9212/1/43253404.2013.pdf

García, V. \& Basilotta, V. (2016). Aprendizaje Basado en Proyectos (ABP): evaluación desde la perspectiva de alumnos de Educación Primaria. Revista de Investigación Educativa, 35(1), 113. Recuperado de http://revistas.um.es/rie/article/view/246811

Jorrín, A, (2016). Evaluando rayuela: una herramienta web para vincular los enfoques paradigmático y pragmático en la enseñanza de métodos de investigación cualitativa.

Larmer, J. \& Mergendoller, J. (2015). Estándares ABP: Características esenciales del aprendizaje basado en proyectos Adapted and translated from Setting the Standard for Project Based Learning: A Proven Approach to Rigorous Classroom Instruction.

Leymonié, J, (2009). Aportes para la enseñanza de las Ciencias Naturales. Director Vol. $3 . \quad$ Recuperado de http://unesdoc.unesco.org/images/0018/001802/180275s.pdf

Martínez, C. (2006). El método de estudio de caso: estrategia metodológica de la investigación científica. Pensamiento \& Gestión, (20), 165-193.

Moreira, M. (1997). Aprendizagem Significativa: um conceito subyacente. En M.A. Moreira, C. Caballero Sahelices y M.L. Rodríguez Palmero, Eds. Actas del II Encuentro Internacional sobre Aprendizaje Significativo. Servicio de Publicaciones. Universidad de Burgos. Págs. 19-44. 
Moreira, M. (2005). Aprendizaje Significativo Crítico. Recuperado de http://www.fbioyf.unr.edu.ar/evirtual/pluginfile.php/131373/mod resource/content/1/apsigcritesp.pdf

Ramírez, D. \& Santana, F. (2014). El aprendizaje basado en proyectos y el aprendizaje de conceptos de calor y temperatura mediante aplicaciones en cerámica. Innovación Educativa, vol. 14(66).

\section{Agradecimientos}

Agradecemos a la Institución Educativa Alfonso Upegui Orozco por su apoyo, colaboración y disposición para la implementación de nuestro proyecto de investigación y a nuestros asesores, Christian Fernney Giraldo Macías y Diana Paola Martínez Salcedo, quienes siempre estuvieron prestos a hacer sugerencias y aportes al presente trabajo. 